\title{
La gubernamentalidad como poder a distancia: Foucault y la crisis de las disciplinas
}

\author{
The Governmentality as a Power at a Distance: Foucault and the \\ Crisis of Disciplines
}

ALFREDO SÁNCHEZ SANTIAGO*

Resumen: Sobre la base de las modificaciones que tienen lugar en la noción de poder de Michel Foucault entre 1975 y 1979 , este trabajo pretende ofrecer algunas claves de análisis de las nuevas modalidades de control político características de las sociedades del capitalismo avanzado. El planteamiento en 1978 de la serie libertad-mecanismos de seguridad-gobierno conduce a Foucault a evacuar el modelo disciplinario como esquema único de inteligibilidad del ejercicio del poder. Queda abierta de esta forma la posibilidad de avanzar en la conceptualización de la gubernamentalidad neoliberal vigente en nuestras sociedades como un poder indirecto o un "gobierno a distancia" que preserva intocada -y declara intocable- la materialidad misma de los cuerpos.

Palabras clave: poder, disciplina, gubernamentalidad, libertad, seguridad, neoliberalismo.

\begin{abstract}
Based on the changes performed on the notion of power in Michel Foucault's discourse from 1975 to 1979 , this work proposes a few elements for the analysis of the most recent methods of political control, specific to late modern societies. The examination in 1978 of the series freedom-mechanisms of security-government leads Foucault to evacuate the disciplinary model as the unique grid of intelligibility for the exercise of power. It thus opens the way to conceptualize current neoliberal governmentality as a form of indirect power or 'government at a distance' which preserves untouched -and declares untouchable- bodies' own materiality.

Keywords: power, discipline, governmentality, freedom, security, neoliberalism.
\end{abstract}

\section{Introducción}

Pocos conceptos en el pensamiento de Foucault ofrecen un rendimiento teórico comparable al de "panoptismo". El término es empleado en Vigilar y castigar para describir la generalización, a finales del siglo XVIII, de una tecnología de poder orientada a la rentabilización económica de las operaciones del cuerpo y al control minucioso de sus capacidades. En virtud de su carácter individualizador y de su aplicación microfísica, Foucault califica

Recibido: 30/01/2017. Aceptado: 27/10/2017.

* Máster en Estudios Avanzados en Filosofía por la Universidad Complutense de Madrid y Máster en Filosofía política y Ética por la Universidad Paris- Sorbonne. Investigador en formación en el Departamento de Filosofía y Sociedad de la UCM (becario UCM-Santander). Líneas de investigación: filosofía política, historia de la filosofía moderna y contemporánea y teoría crítica de la sociedad. Publicaciones recientes: "Aproximación al concepto de genealogía en Nietzsche y Foucault", La Torre del Virrey: Revista de Estudios Culturales, $\mathrm{n}^{\circ} 22$, 2017/2. Contacto: alfredo.sanchez@ucm.es. 
este poder como "disciplinario", y ofrece de él una descripción que toma como punto de partida los efectos que asegura sobre los cuerpos individuales: sujeción interna, distribución espacial, organización jerárquica, control temporal de las actividades, composición de las fuerzas en función de tareas específicas, homogeneización de los comportamientos por referencia a una norma preestablecida. Incorporado desde el siglo anterior en espacios institucionales cerrados (escuelas, talleres, hospitales y prisiones), el "poder disciplinario" se habría difundido en los primeros tiempos del capitalismo como un mecanismo compacto e indefinidamente generalizable de control político, pero también como el instrumento activo de un poder encaminado a fabricar la fuerza productiva requerida por el nuevo aparato de producción: "para que haya plusganancia -resume Foucault- es preciso que haya subpoder" (Foucault, 2011, 146). De acuerdo con el diagnóstico foucaultiano de la primera mitad de los años setenta, la sociedad contemporánea constituiría la última materialización histórica del panoptismo, la confirmación práctica del proyecto utópico de Bentham. Las palabras pronunciadas por Foucault en las conferencias de Río de Janeiro del año 1973 no dejan lugar para la ambigüedad: "La sociedad contemporánea puede ser denominada [...] sociedad disciplinaria. [...] El panóptico, explica Foucault, es la utopía de una sociedad y un tipo de poder que es, en el fondo, una utopía que efectivamente se realizó. Este tipo de poder bien puede recibir el nombre de 'panoptismo': vivimos en una sociedad en la que reina el panoptismo" (Foucault, 2011, 95).

Algunos años después, en abril de 1978, Foucault concede en el instituto francés de Kansai, en Kyoto, una entrevista que aparecerá publicada bajo el título significativo de "La société disciplinaire en crise". Interrogado acerca de la posibilidad de hacer extensibles sus análisis sobre el poder al caso particular de la sociedad japonesa, Foucault completa su respuesta en una dirección inesperada: "La disciplina, que era tan eficaz para mantener el poder, ha perdido una parte de su eficacia. En los países industrializados, las disciplinas entran en crisis". La digresión no pasa desapercibida para su interlocutor, que no duda en preguntarle sobre el significado de esta crisis de la sociedad disciplinaria. A lo que Foucault responde: "Estos últimos años, la sociedad ha cambiado y también los individuos, que son cada vez más diversos, diferentes e independientes. Cada vez existen más categorías de personas que no se encuentran constreñidas por las disciplinas, por lo que estamos obligados a pensar el desarrollo de una sociedad sin disciplina" (Foucault, 1994, 533).

Entre uno y otro escenario, lo que ha variado es el acercamiento de Foucault a la tradición intelectual del neoliberalismo, a cuyo estudio dedica en 1979 su lección anual en el Collège de France. Con la racionalidad de gobierno neoliberal, Foucault se ve confrontado a un programa de reforma de la sociedad y del individuo que, en contraste con el modelo disciplinario, privilegia la diversidad (de las empresas individuales) sobre la homogeneización, la optimización de la diferencia y la tolerancia de las desviaciones individuales sobre la estandarización de los comportamientos, la independencia de los individuos en detrimento de su subordinación a los distintos aparatos institucionales, la conducción a distancia de sus conductas sobre la dominación microfísica de sus gestos y de sus operaciones. La apertura del problema de la libertad, planteado por vez primera en el curso del año 1978 sobre el arte liberal de gobernar, constituye el elemento detonante del retroceso progresivo de las disciplinas como principio arquitectónico de la analítica foucaultiana y como esquema único de inteligibilidad del ejercicio de poder en nuestras sociedades. 
En las líneas que siguen querríamos ocuparnos de este repliegue de lo disciplinario, contribuyendo así a sistematizar una de las perspectivas desde las que es posible acercarse a la producción foucaultiana de finales de la década de los setenta. Pero de los cursos de Foucault sobre la racionalidad liberal y neoliberal de gobierno creemos posible extraer una segunda lectura, a la que subordinamos la contribución fundamental de este trabajo. Pensamos que en estas lecciones puede leerse, además del retroceso de lo disciplinario (o como su correlato teórico), el esfuerzo de Foucault por conceptualizar una modalidad de poder "a distancia" que interviene sobre el medio de acción de los individuos, y que funciona habilitando espacios de libertad, disponiendo márgenes de tolerancia, articulando acciones ambientales, flexibilizando normas de acción, reemplazando la regla uniforme y dirigista de las disciplinas por un modo de gobierno basado en preceptos elásticos, abandonando, en fin, la materialidad misma de los cuerpos como objeto prioritario de intervención política. Tomando pie, por lo tanto, en algunas de las últimas reflexiones de Foucault sobre el poder, se trataría de proyectar ciertos elementos de análisis de esta racionalidad mesológica de dominación que creemos vigente en nuestras sociedades, y cuya dinámica interna resulta inaprehensible a partir del viejo aparato conceptual ofrecido por el modelo de las disciplinas, pues presupone precisamente su agotamiento.

El concepto de gubernamentalidad vehicula adecuadamente el sentido de este movimiento correlativo del pensamiento foucaultiano ocurrido a finales de los años setenta: retroceso de lo disciplinario - conceptualización de un modelo de gobierno a distancia. Presentada como una noción "problemática y artificial", "difusa" y "fragmentaria" (Foucault, 2009a, 140), la gubernamentalidad pasará a ocupar un lugar destacado en el repertorio filosófico de Foucault, convirtiéndose desde 1978 en el nuevo marco teórico de su genealogía del poder. Esta noción constituye el objeto del capítulo que sigue, en el que tratamos de dar cuenta de su complejidad presentando las diversas acepciones que reviste en las lecciones de los años 1978-1979.

\section{Sobre la polisemia de la noción de gubernamentalidad}

En Seguridad, territorio, población (1977-1978) y en Nacimiento de la biopolítica (1978-1979), el concepto de gubernamentalidad se emplea con sentidos diversos que unas veces se complementan, y otras se solapan o se contradicen. Sin afán de exhaustividad y por centrar en la medida de lo posible la serie de problemas que tratan estos cursos, nosotros proponemos cuatro:

1) En primer lugar, la noción de gubernamentalidad ofrece una herramienta metodológica que permite remitir el problema del Estado al dominio general de las relaciones de poder $^{1}, \mathrm{y}$, en concreto, al conjunto de las prácticas de gobierno que han asegurado de hecho su consistencia, su desarrollo y sus mutaciones a lo largo de la historia. Como las disciplinas, la gubernamentalidad constituye un punto de vista (Foucault, 2009b, 188): el de las relaciones de poder, pero ampliado en esta ocasión a un nuevo objeto (no la prisión o la fábrica, sino el Estado) que no había sido tratado más que de un modo marginal en la

1 Seguimos en esta cuestión algunas de las observaciones de Michel Senellart en su "Situación de los cursos" (Foucault, 2009a, pp. 417-453) y de Thomas Lemke (2004). 
obra foucaultiana precedente. De lo que se trata es de negar al Estado toda sustancialidad, de adoptar con respecto a él un punto de vista externo que haga posible señalar las prácticas múltiples de gubernamentalidad de las que depende y a través de las cuales conforma en cada momento su singular apariencia histórica: el Estado, afirma Foucault, constituye así una realidad compuesta, la condensación siempre inestable de redes heterogéneas de poder, el "efecto móvil de un régimen de gubernamentalidades múltiples" (Foucault, 2009b, 84).

2) Esta consideración externalista del Estado habilita a Foucault para poner en tela de juicio una cierta corriente de "fobia al Estado" que, desde los años treinta del siglo XX, puede ser detectada en algunos sectores de la crítica social y política. Auspiciada por los teóricos neoliberales de la Escuela de Friburgo (F. Böhm, W. Eucken, A. Müller-Armack, W. Röpke, A. Rüstow, entre otros), esta crítica se funda sobre dos supuestos: en primer lugar, sobre la idea de que al Estado corresponde una tendencia indefinida de expansión que lo conduciría a invadir los espacios de libertad en los que se desarrolla la iniciativa privada de los individuos. En segundo lugar, sobre el reconocimiento de una continuidad ontológica entre formas estatales tan diversas como el Estado administrativo, el Estado benefactor, el Estado burocrático o el Estado fascista.

Resulta llamativa la contundencia con la que Foucault descalifica esta crítica generalizada del Estado y de su carácter supuestamente omnipresente, violento y polimorfo ${ }^{2}$. Además de su imprecisión y de su inespecificidad, Foucault denuncia el carácter extemporáneo de estos análisis: "lo que hoy está en cuestión en nuestra realidad no es tanto el crecimiento del Estado y la razón de Estado sino más bien, y mucho más, su decrecimiento" (Foucault, 2009b, 193). Pues bien -y este es el aspecto que nos interesa resaltar-, la noción de gubernamentalidad desempeña aquí una segunda función significativa. Según expone Foucault, el proceso que define adecuadamente la ontología política de nuestro presente no es tanto la estatización de la sociedad cuanto la "“gubernamentalización’ del Estado" (Foucault, 2009a, 137; Bidet, 2006, 172), o, lo que es igual, la disolución de su consistencia jurídico-política en tácticas múltiples y heterogéneas de gestión económica de una población. La función metodológica de la noción de gubernamentalidad, que permitía comprender el Estado como el producto de diversas operaciones de gobierno, entronca en este punto con una función crítica que deja al descubierto el carácter "inflacionario" de los ataques que se le dirigen. Aprehendiendo genealógicamente cada una de sus configuraciones, haciéndolo aparecer como el efecto de decisiones concretas de gobierno, se evita conceder al Estado una importancia, una unidad y una funcionalidad rigurosa que, ni tiene en la actualidad, ni tuvo a lo largo de su historia (Foucault, 2009a, 136-137).

3) En tercer lugar, la noción de gubernamentalidad consuma la ampliación del foco de la investigación desde el estudio de la disciplina de los cuerpos hacia el de la regulación biopolítica de las poblaciones. Mediante ella y en continuidad con el curso del año 1976, Foucault trata de determinar el dominio estratégico de relaciones de poder en el que se juega el ejercicio del poder biopolítico. En la que quizá constituya una de sus formulaciones más

2 Al menos si tenemos en cuenta la conocida disposición anti-institucional de sus escritos publicados a comienzos de la década de los setenta. A modo de ejemplo, basten algunas líneas del manifiesto fundacional del Grupo de Información sobre las Prisiones, que lleva por título «Intolerable»: «Son intolerables: los tribunales, la bofia [les flics], los hospitales, los manicomios, la escuela, el servicio militar, la prensa, la tele, el Estado y en primer lugar las prisiones» (cit. en Eribon, 1992, 275). 
oscuras, la gubernamentalidad es definida en Seguridad, territorio, población como "el conjunto constituido por las instituciones, los procedimientos, análisis y reflexiones, los cálculos y las tácticas que permiten ejercer esa forma bien específica, aunque muy compleja, de poder que tiene por blanco principal la población, por forma mayor de saber la economía política y por instrumento técnico esencial los dispositivos de seguridad" (Foucault, 2009a, 136).

Adviértase que la gubernamentalidad no es presentada en esta fórmula como un tipo de poder, sino como el conjunto de saberes y de prácticas socio-políticas que permiten explicar el ejercicio de esa forma de poder que es la biopolítica. Pensamos que esta idea puede ser comprendida a la luz de lo planteado dos años antes en el curso Hay que defender la sociedad (1976): en él se afirmaba que, a diferencia de las disciplinas y en razón de la complejidad de las regulaciones globales que involucra, la gestión biopolítica de las masas humanas necesita de "órganos complejos de coordinación y centralización" que es preciso buscar por el lado del Estado. En este sentido, Foucault planteaba dos series: "la serie cuerpo - organismo - disciplina - instituciones; y la serie población - procesos biológicos - mecanismos regularizadores - Estado" (Foucault, 2003, 214).

Se impone entonces una pregunta como la siguiente: el ejercicio del poder biopolítico, ¿es posible gracias a la gubernamentalidad (como se afirma en 1978) o más bien al aparato de Estado (como sostiene Foucault dos años antes)? En coherencia con la primera función de la gubernamentalidad antes mencionada (hacer entrar al Estado dentro del campo de análisis de los micropoderes), sólo cabe responder algo como lo siguiente: el Estado es aquello que permite el ejercicio del poder biopolítico, a condición de entender por Estado un efecto de gubernamentalidades múltiples, esto es, un conjunto históricamente determinado de instituciones, procedimientos, análisis, reflexiones, cálculos y tácticas. En una palabra, a condición de comprender el Estado como una "práctica" (Foucault, 2009a, 324)

4) Ahora bien, la gubernamentalidad designa en los cursos de 1978-1979 también un tipo de poder, y no sólo el entramado de saberes y de prácticas que hacen posible su ejercicio biopolítico. En tanto que tecnología política, el concepto de gubernamentalidad se vuelve prácticamente indiscernible del de gobierno, entendido en un sentido muy amplio como "la manera de conducir la conducta de los hombres" (Foucault, 2009b, 188). La comprensión bélica de la política, que había inspirado otros textos anteriores, planteaba una lucha cuerpo a cuerpo entre el poder y los individuos, cuya iniciativa quedaba anulada por una dominación microfísica dirigida hacia sus gestos, sus actos y sus operaciones (López Álvarez, 2006). Con el gobierno, Foucault transita hacia un tipo de poder con respecto al cual los individuos, sin caer presos por completo de la dominación, son conducidos mediante regulaciones generales en tanto que sujetos capaces de acción y de respuesta.

En uno de sus últimos escritos, publicado en 1983, Foucault explica que con la noción de gobierno pretende designar "un modo de acción que no actúa de manera directa e inmediata sobre los otros, sino que actúa sobre sus acciones: una acción sobre la acción, sobre acciones eventuales o actuales, presentes o futuras" (Foucault, 1988, 238). La intervención del poder, por tanto, no apunta al "centro" de los individuos sino -por así decir- a su "periferia", no a sus cuerpos sino a lo que los individuos hacen o están interesados en hacer. La acción de gobierno presupone en los gobernados una cierta actividad, un margen suficientemente holgado para el ejercicio de la libertad: "El poder se ejerce únicamente sobre 'sujetos libres' y sólo en la medida en que son libres. Por esto queremos decir sujetos individuales 
o colectivos, enfrentados con un campo de posibilidades, donde pueden tener lugar diversas conductas, diversas reacciones y diversos comportamientos" (Foucault, 1988, 239). En definitiva, el gobierno "deja hacer" a los individuos, sobre cuyas acciones ejerce, no una reglamentación inflexible, como era el caso de la disciplina -que "no deja escapar nada" (Foucault, 2009a, 67)-, sino una labor general de dirección [guidage].

Como tendremos ocasión de comprobar en los capítulos que siguen, el deslizamiento de la investigación hacia la cuestión del gobierno conlleva, por lo pronto, el abandono de hecho de las disciplinas como modelo privilegiado de interpretación del ejercicio del poder, o, lo que es igual, la sustitución de la dominación de los cuerpos por la conducción de las conductas como gramática fundamental de la relación política. Pero, además y como consecuencia, supone la primera tentativa de Foucault para describir en toda su complejidad la relación entre poder y libertad, relación que su obra anterior no permitía pensar sino de un modo negativo ${ }^{3}$. En efecto, el díptico constituido por los cursos de los años 1978-1979 revela los usos técnico-políticos de la libertad, y, en esta medida, permite a Foucault transitar hacia la consideración de una nueva modalidad de poder que no precisa para su ejercicio de la coacción directa ni del contacto físico con los individuos: un poder a distancia, una intervención exhaustiva sobre las reglas del juego que preserva intocados -y declara intocables- a sus jugadores.

\section{La gubernamentalidad liberal o sobre la posibilidad de una acción política a distancia}

Ha señalado Frédéric Gros que Foucault no estudia el liberalismo como doctrina ni como ideología, sino como gubernamentalidad política, es decir, como arte de conducción de las conductas de los individuos (Gros, 2013). El liberalismo es comprendido como un estilo de gobierno dotado de reglas, instrumentos y objetos específicos, pero ante todo como un "operador crítico" que provoca en el seno de la soberanía el siguiente interrogante: "¿gobierno bien en el límite de lo demasiado y lo demasiado poco, entre ese máximo y ese mínimo que me fija la naturaleza de las cosas, esto es, las necesidades intrínsecas a las operaciones de gobierno?" (Foucault, 2009b, 31-32). El gobierno liberal, sentencia Foucault, es un "gobierno frugal" (Foucault, 2009b, 38) cuyo principio de limitación le viene impuesto por la naturalidad de sus propios mecanismos, pero también por la naturalidad de los objetos que conforman el ámbito de su intervención. ¿De qué objetos naturales se trata y en qué consiste su naturalidad?

En primer lugar, se trata del mercado, que se erige en "lugar de veridicción" (Foucault, 2009b, 41) de la práctica gubernamental en la medida en que del conocimiento y el respeto de sus mecanismos espontáneos y de sus leyes naturales depende la corrección o incorrección de aquella. En segundo lugar, se trata de la población, que está tan sometida como la riqueza a leyes naturales de desplazamiento, crecimiento y transformación, así como a toda una serie de variables materiales: clima, comercio, costumbres, disponibilidad de artículos de subsistencia, valores morales o religiosos (Foucault, 2009a, 93).

3 Un estudio pormenorizado de la articulación entre poder y libertad a lo largo de la analítica foucaultiana puede encontrarse en Castro Orellana (2006). 
Detengámonos un momento en esta caracterización de la "población" como fenómeno natural. En virtud de los procesos naturales que la atraviesan y de los mecanismos internos que espontáneamente la regulan, la población es presentada como un fenómeno refractario a una intervención legalista o disciplinaria que proyecte sobre ella un sistema reglamentario de imperativos, prohibiciones y mandatos. La idea de Foucault es que la población sólo será permeable a la práctica de gobierno si, en lugar de actuar directamente sobre ella, se opta por intervenir sobre "una multitud de factores, elementos que en apariencia están lejos de la población misma y su comportamiento inmediato, lejos de su fertilidad, de su voluntad de reproducción" (Foucault, 2009a, 95, cursiva nuestra). La población, objeto de la práctica de gobierno, se vuelve penetrable por esta última desde el momento en que su control no se asegura mediante una acción directa, sino a través de una acción a distancia dirigida al medio del que la población depende. Operando sobre las distintas variables del medio, el gobierno liberal es capaz de actuar indirectamente sobre la población, de favorecer sus actividades productivas, de modificar sus tendencias indeseables, de minimizar sus circulaciones nocivas. Casi sin atender al detalle de cada cuerpo, prescindiendo, por lo tanto, de una aplicación irrestricta del poder disciplinario, el gobierno tampoco dirige su intervención hacia la población, o no lo hace más que lateralmente, pues su nuevo correlato lo constituye el conjunto de datos materiales que actúa sobre los individuos y sobre el cual, recíprocamente, ellos también actúan: "se ve aparecer con toda claridad la noción de un medio histórico natural como blanco de una intervención de poder, que me parece muy diferente de la noción jurídica de la soberanía y el territorio y también del espacio disciplinario" (Foucault, 2009a, 42, cursiva nuestra).

De acuerdo con el paradigma liberal, existe una segunda manera de que la población pueda volverse manejable por la acción del gobierno: operar sobre el interés que determina naturalmente la acción de los individuos. El punto de contacto entre el poder y la población es en esta ocasión el interés del individuo, vale decir del homo oeconomicus: dejándolo actuar según sus propias leyes, liberándolo de todas las restricciones que pudieran interferir en sus actividades, es razonable esperar que su interés privado acabe redundando en el beneficio colectivo de la población. Como en el caso anterior, el poder liberal se desprende de la tendencia legalista y disciplinaria que había caracterizado al Polizeistaat del siglo XVII, abandona esas "cosas en sí" que son los sujetos individuales para concentrarse en sus intereses, en esa especie de fenómenos "exteriores" al cuerpo de los individuos que, manejados como corresponde, contenidos en ciertos límites, estimulados también cuando es preciso permiten un control aceptable de las multitudes. Los intereses, explica Foucault, son "el medio por el cual el gobierno puede tener influjo sobre todas esas cosas que para él son los individuos, los actos, las palabras, las riquezas, los recursos, la propiedad, los derechos, etcétera" (Foucault, 2009b, 56, cursiva nuestra).

En su trato con el medio material y con los intereses individuales, el poder liberal exhibe una clara disposición biopolítica, pues se muestra capaz de recuperar un fenómeno natural en el espacio artificial de una relación de poder (Foucault, 2009a, 42). Pero de este desarrollo se puede extraer una segunda consecuencia, ligada a la anterior: la concepción de lo que ya hemos calificado como una racionalidad mesológica de gobierno de los hombres, y el retroceso correlativo de las disciplinas como instrumento técnico-político fundamental de la modalidad liberal de gobierno. La distinción entre mecanismos disciplinarios y dispositivos 
de seguridad, que ya había provocado ciertas variaciones teóricas en el curso del año 1976, adquiere en este momento su más intensa significación. Los dispositivos de seguridad, y no tanto las disciplinas, aparecen como la técnica predominante de la economía de poder liberal, como puede constatarse en los siguientes tres ámbitos:

i) En primer lugar, en el tratamiento que el liberalismo hace del espacio: mientras que las disciplinas tienen un carácter centrípeto consistente en circunscribir un espacio artificial, vacío y cerrado, los dispositivos de seguridad poseen un carácter centrífugo, pues su objetivo es concebir espacios cada vez más amplios que permitan la circulación de las masas y de las mercancías (Foucault, 2009a, 36).

ii) En segundo lugar, si las disciplinas tenían por función la reglamentación detallada de los individuos, la seguridad inserta el acontecimiento que se quiere regular en un cálculo de costos que permite fijar "por una parte una media considerable como óptima y por otra límites de lo aceptable, más allá de los cuales ya no habrá que pasar" (Foucault, 2009a, 21).

iii) De ahí, por último, el tipo de normalización que aplican los dispositivos de seguridad en la razón liberal de gobierno, menos interesada en clasificar los fenómenos de acuerdo con la distinción disciplinaria entre lo normal y lo anormal que en aproximar, a partir de un análisis minucioso de los tipos de normalidad posibles, las normalidades más desfavorables a las más favorables (Foucault, 2009a, 83).

Sobre la base de estas distinciones -conviene insistir en este punto-, Foucault puede corregir su anterior diagnóstico sobre la conexión entre la libertad y los dispositivos de poder (López Álvarez, 2010, 44-46). Definido el arte liberal de gobernar como un principio interno de limitación del poder, un gobierno frugal, un poder sobre el medio, una práctica gubernamental caracterizada por la flexibilidad de las técnicas políticas que emplea, parece difícil pensar que pudiera albergar en sí un conflicto entre las técnicas de poder que articula y los ámbitos de libertad que se ve obligado a producir. Los textos de mediados de los setenta presentaban las técnicas de control de las sociedades como procedimientos limitadores de la libertad de los individuos, la cual era comprendida a su vez como un artefacto ideológico que permitía encubrir el oscuro sistema de dominación panóptica en el que se encontraba atrapada la vida de los hombres. La célebre afirmación de Foucault en Vigilar y castigar: "Las Luces, que han descubierto las libertades, inventaron también las disciplinas" (Foucault, 1975/2012a), es modulada ahora en los siguientes términos:

[...] en alguna parte dije que no se podía comprender la introducción de las ideologías y una política liberales en el siglo XVIII sin tener presente que esa misma centuria que había reivindicado en tan alta voz las libertades las había lastrado, empero, con una técnica disciplinaria que [...] limitaba en forma considerable la libertad y daba en cierto modo garantías a su ejercicio. Pues bien, creo que me equivoqué. No me equivoqué por completo, por supuesto, pero en fin, no es exactamente así. Lo que está en juego, me parece, es muy otra cosa. Esa libertad, a la vez ideología y técnica de gobierno, debe comprenderse en el interior de las mutaciones y transformaciones de las tecnologías de poder. Y de una manera más precisa y particular, la libertad no es otra cosa que el correlato de la introducción de los dispositivos de seguridad (Foucault, 2009a, 71). 
La imagen de la cuadrícula disciplinaria, que había servido a Foucault durante algunos años para representar los mecanismos de poder en sus dimensiones más ínfimas, sus combinaciones recíprocas y sus alternancias, resulta, de ahora en adelante, demasiado estrecha para enmarcar un modo de circulación del poder en el que la libertad aparece necesariamente convocada. Entre el individuo y el poder, las disciplinas cubren todos los espacios, neutralizando cualquier posibilidad de resistencia o convirtiendo cada pretendida liberación en una nueva modalidad -más compleja y refinada, si cabe-de sometimiento. En cambio, en el modelo de gobierno no cabe concebir poder sin libertad ni libertad sin poder: entre el poder y el individuo, hay una distancia que debe ser mantenida, no por el respeto debido a ciertos derechos inalienables del hombre, sino porque de dicha distancia depende el éxito de la acción misma de gobierno.

La libertad, entendida en un sentido muy amplio como "posibilidad de movimiento, desplazamiento, proceso de circulación de la gente y las cosas" (Foucault, 2009a, 71), es decir, como libertad económica, es el correlato de los dispositivos de seguridad y, por ende, de la razón liberal de gobierno. Pensada en la microfísica del poder de mediados de los setenta como un epifenómeno de superficie, la libertad queda integrada en lo sucesivo en el campo operativo de las tecnologías de poder: no como un dato previo o un derecho originario que debe ser respetado, sino como aquello que tiene que ser producido a cada instante para salvaguardar la verdad (el éxito o la utilidad, y no la legitimidad) de la acción misma de gobierno. El gobierno liberal, como también el neoliberal, mantienen una relación de seducción e incitación permanentes con la libertad, que constituye al mismo tiempo su condición, su producción y su objeto de consumo (Díaz Marsá, 2014, 38; Sardinha, 2005).

\section{El individuo intocable o sobre la política ambiental del neoliberalismo}

Las tesis presentadas en Nacimiento de la biopolítica terminan de socavar los cimientos del paradigma disciplinario, desencadenando su retroceso masivo, y prefiguran algunos rasgos del nuevo tipo de poder postpanóptico que se encuentra vigente en nuestras sociedades.

Debido, por una parte, a su vinculación con los problemas de nuestra actualidad más inmediata, y, por otra, a la habitual e incómoda neutralidad axiológica del discurso de Foucault, este curso ha suscitado reacciones divergentes en el panorama filosófico de los estudios foucaultianos. Algunos comentaristas han visto en él el acercamiento de Foucault a posiciones políticas cercanas al liberalismo y al neoliberalismo, cuyos programas de constitución de subjetividades no normalizadas habrían ejercido un profundo poder de seducción sobre el pensador francés (Moreno Pestaña, 2010/2011). En la misma línea, se ha escrito acerca de la fascinación con la que Foucault habría acogido la pluralidad, la incoherencia y el carácter fragmentario que está en la base de la concepción neoliberal del mundo social, así como la apuesta del neoliberalismo por una política de las singularidades marcadamente anti-estatista, en la que habría visto la encarnación contemporánea de la teoría crítica y la única esperanza de la tradición emancipadora (Lagasnerie, 2012). En el extremo opuesto, otras lecturas han interpretado el curso del año 1978-1979 como la rehabilitación por parte de Foucault de un cierto pensamiento de lo jurídico-estatal, que contrastaría notablemente con la clara disposición anti-institucional que exhibían algunos de sus textos más conocidos de comienzos de la década de los setenta (Díaz Marsá, 2014). 
No se encuentra entre los objetivos de este trabajo ocuparse de esta cuestión, y mucho menos dirimir la controversia acerca de las variaciones ideológicas del último Foucault. En la línea de lo que ya ha sido expuesto, nuestro interés en este capítulo consiste en delimitar más ajustadamente el proceso correlativo de regresión de lo disciplinario y de despliegue de una modalidad de poder a distancia que sería característico de las sociedades del capitalismo avanzado, poniendo esta vez el foco en la lectura que hace Foucault del derecho penal neoliberal.

Sin duda, el problema de la penalidad proporciona a Foucault un ventajoso punto de vista para percibir la modalidad específica de poder hacia la que el neoliberalismo apunta, pues constituye en toda sociedad un espacio especialmente denso de concentración de relaciones de poder. Esta era, en todo caso, la convicción de Foucault en 1972, quien se pregunta lo siguiente en conversación con Deleuze: “¿No ocurrirá que, de un modo general, el sistema penal es la forma en la que el poder en tanto que poder se muestra del modo más manifiesto?" (Foucault, 2000, 11). Existen suficientes razones para suponer que, a finales de la década de los setenta, Foucault sigue dando por válido este argumento. Resulta significativo a este respecto que, en la primera clase de Seguridad, territorio, población, sea precisamente la penalidad el problema escogido para ejemplificar la diferencia entre las economías de poder basadas en el paradigma de la ley, la disciplina y la seguridad (Foucault, 2009a, 19-25).

Según interpreta Foucault, los análisis de la criminalidad y la justicia penal llevados a cabo por el anarcocapitalismo norteamericano (Gary Becker, Isaac Ehrlich y George J. Stigler) conducen hasta sus últimas consecuencias uno de los presupuestos fundamentales del pensamiento neoliberal: la extensión de la forma del mercado a procesos, relaciones y comportamientos prima facie no económicos. Como ha señalado Antoine Garapon (Garapon, 2010, 113-114), el neoliberalismo establece el discurso de la economía, y no el del derecho positivo o el de las ciencias humanas, como régimen de veridicción y principio de inteligibilidad de la práctica judicial en materia penal, y lo hace de dos maneras distintas:

Por un lado, en continuidad con los reformadores del sistema judicial y penal del liberalismo clásico (Bentham, Beccaria, Brissot), los teóricos neoliberales abordan la cuestión de la penalidad a partir de un cálculo de utilidad, interrogándola en los términos de una economía política: ¿cuál es el coste de la delincuencia? ¿Cuál el de la práctica y las instituciones judiciales? ¿Es más costosa para la sociedad una represión severa o una represión laxa, un castigo ejemplar y discontinuo o una represión continua que incida sobre el más mínimo de los detalles? ¿Cuál de ellas es más eficaz?

Por otro lado, y en esto se distinguen de los grandes reformadores, los neoliberales norteamericanos tratan de contener el análisis de la criminalidad en los límites estrictos de una problemática de tipo económico, evitando los deslizamientos teóricos que finalmente acabó provocando la reforma del derecho penal en el siglo XVIII: especialmente, el que se produjo entre el homo penalis y el homo criminalis, entre el individuo en tanto que sujeto penalizable y el individuo en tanto que sujeto cuyo crimen debe ser dilucidado por medio de toda una inflación de saber psicológico, sociológico y antropológico. De acuerdo con la lectura de Foucault, el neoliberalismo norteamericano lleva a cabo la "borradura antropológica del criminal" (Foucault, 2009b, 259), que deja de ser interrogado sobre la base de rasgos morales y antropológicos y pasa a ser comprendido como un homo oeconomicus, esto es, como una persona cualquiera "que invierte en una acción, espera de ella una ganancia y acepta el riesgo 
de una pérdida" (Foucault, 2009b, 252). Tratando al criminal como un ser racional capaz de tomar decisiones, de efectuar transacciones y de realizar un cálculo de interés (Garapon, 2013, 44), el discurso neoliberal desmoraliza a los individuos, pero proponiendo al mismo tiempo -como ha señalado Émilie Hache- "un nuevo discurso moralizante, organizado en torno a la idea de conducta "responsable"" (Hache, 2007, 58, trad. nuestra). Como en otros ámbitos de la racionalidad neoliberal ${ }^{4}$, el individuo es impelido por el nuevo modelo de penalidad a conducirse como un individuo autorresponsable, obligado no sólo a realizar una evaluación de los riesgos antes de pasar al acto, sino a asumir todas y cada una de las consecuencias que puedan seguirse de una conducta no racional, resultado de un mal cálculo.

Lo anterior nos sitúa ante una innovación esencial del neoliberalismo como práctica de gobierno de los individuos. En la medida en que su comportamiento admite ser interpretado según una clave de análisis estrictamente económica, el individuo criminal se vuelve "gubernamentalizable", dice Foucault. Ahora bien -y esta es la cuestión que más nos interesa subrayar-, que la individualidad criminal sea gobernable no quiere decir en modo alguno que constituya el blanco del poder de identificación, de uniformización y de individualización de las disciplinas. De nuevo, el individuo es más bien el objeto de una conducción laxa y a distancia, de un tipo de gubernamentalidad mesológica que busca influir sobre su comportamiento desviando la trayectoria de sus intervenciones hacia el medio de acción en el que el individuo opera: "el homo oeconomicus pasa a mostrarse ahora como el correlato de una gubernamentalidad que va a actuar sobre el medio y modificar sistemáticamente sus variables" (Foucault, 2009b, 67).

¿De qué modo concreto aplica la justicia penal neoliberal este modelo de control político? A través de una redefinición muy singular de la función de la ley. Explica Denis Salas (Salas, 2012, 64-66) que el derecho penal neoliberal define la ley como una información que, en la medida en que se presenta como fiable y previsible, permite al criminal potencial realizar una evaluación de los riesgos, y a las instituciones prevenir los actos criminales y disuadir a sus autores de cometerlos. Con arreglo al análisis de Foucault, la ley se limita a establecer las "reglas del juego", acondicionando "el medio del mercado en el que el individuo hace su oferta de crimen" (Foucault, 2009b, 260, cursiva nuestra) y oponiendo a esta oferta una demanda negativa. La ley es elástica porque admite ser modulada de acuerdo con un cálculo de utilidad, como ya hemos visto, pero también porque su intervención debe limitarse a "la definición en torno del individuo de un marco lo bastante laxo para que él pueda jugar" (Foucault, 2009b, 262), desarrollar sus acciones y sus iniciativas. La acción penal, en definitiva, debe definir tan solo una acción ambiental sobre el juego de las ganancias y las pérdidas posibles: "si no se quiere salir de la ley y desvirtuar su verdadera función de regla del juego, la tecnología que será menester utilizar no es la disciplina-normalización, es la acción sobre el ambiente. Modificar el reparto de las cartas del juego, no la mentalidad de los jugadores" (Foucault, 2009b, 261-262) ${ }^{5}$, concluye Foucault.

4 Encontramos dos buenos ejemplos en la esfera del trabajo, con la figura del emprendedor o empresario de sí mismo, y en la de las políticas sociales, con el desmantelamiento de las medidas asistenciales características del Estado social de Bienestar en favor de dispositivos de cobertura individual de los riesgos (cfr. Dardot y Laval, 2009, 314).

5 Sobre esta cuestión, remitimos al lector al trabajo de Taylan (2013). 
De lo anterior se pueden extraer al menos dos consecuencias, que presentamos ya de forma muy esquemática. En primer lugar, la relajación del cerco de control político que se establece en torno al individuo. Más cercano al modelo de la seguridad que al de la normalización disciplinaria, el derecho penal del neoliberalismo deja intocado el cuerpo de los individuos para intervenir sobre el medio en el que, como sujetos económicos, persiguen libremente la realización de sus intereses privados. En segundo lugar, la renuncia al ideal disciplinario de un control total sobre los cuerpos. En la medida en que se limita a una intervención periférica sobre la esfera de los intereses del individuo criminal, el neoliberalismo no aspira, no puede aspirar, a la extinción total del crimen, sino al establecimiento de un equilibrio razonable entre oferta de crimen y demanda negativa: "La sociedad -explica Foucault- no tiene necesidad alguna de obedecer a un sistema disciplinario exhaustivo. Una sociedad está cómoda con cierto índice de ilegalidad y estaría muy mal si quisiera reducirlo indefinidamente" (Foucault, 2009b, 256-257).

En definitiva, los desarrollos precedentes parecen convencer a Foucault de la necesidad de emparentar el gobierno neoliberal con una economía de poder basada en el orden de la seguridad (gubernamentalidad mesológica, cálculo de costos, aceptación de la libertad, permisividad, fijación de límites de lo aceptable). El abandono del modelo disciplinario es la consecuencia necesaria:

[...] lo que aparece en el horizonte de un análisis como éste no es en modo alguno el ideal o el proyecto de una sociedad exhaustivamente disciplinaria en que la red legal que ciñe a los individuos sea relevada y prolongada desde adentro por mecanismos, digamos, normativos. No es tampoco una sociedad en la que se exija el mecanismo de la normalización general y la exclusión de lo no normalizable. En el horizonte de ese análisis tenemos, por el contrario, la imagen, la idea o el tema-programa de una sociedad en la que haya una optimización de los sistemas de diferencia, en la que se deje campo libre a los procesos oscilatorios, en la que se conceda tolerancia a los individuos y las prácticas minoritarias, en la que haya una acción no sobre los participantes en el juego, sino sobre las reglas de éste, y, para terminar, en la que haya una intervención que no sea del tipo de la sujeción interna de los individuos, sino de tipo ambiental (Foucault, 2009b, 261).

En una hoja no paginada de su manuscrito del curso, de acuerdo con las indicaciones del editor, Foucault resume así el sentido general de estos desplazamientos: "retroceso masivo con respecto al sistema normativo disciplinario" (Foucault, 2009b, 261).

\section{Epílogo: postpanoptismo y sociedades de seguridad}

Podrá objetarse que nuestro planteamiento sobredimensiona la oposición entre disciplina y seguridad como modelos de análisis de las relaciones de poder, oposición que el propio Foucault matiza, efectivamente, en la primera sesión del curso Seguridad, territorio, población: 
No hay era de lo legal, era de lo disciplinario, era de la seguridad. No tenemos mecanismos de seguridad que tomen el lugar de los mecanismos disciplinarios, que a su vez hayan tomado el lugar de los mecanismos jurídico legales.

Esto es cierto, como también lo es que, justo a continuación e igualmente en otros lugares, Foucault se refiere a la posibilidad de señalar, en la economía general de poder de una sociedad, una técnica dominante: "[...] lo que va a cambiar es sobre todo la [técnica] dominante, o más exactamente, el sistema de correlación entre los mecanismos jurídico legales, los mecanismos disciplinarios y los mecanismos de seguridad" (Foucault, 2009a, 23). Y es la pregunta acerca de si la seguridad constituye la técnica dominante en la economía de poder vigente en nuestras sociedades la que parece dirigir en algún momento la reflexión de Foucault en el curso del 78: “¿podemos decir que en nuestras sociedades la economía general de poder está pasando a ser del orden de la seguridad?" (Foucault, 2009a, 26).

Pensamos que los cursos de 1978-1979 dejan de lado las disciplinas para concentrarse en la seguridad, y que los fragmentos de ambas lecciones dedicados a salvaguardar su interrelación en las distintas versiones de los gobiernos liberales resultan considerablemente vagos e imprecisos, además de poco numerosos (Foucault, 2009a, 21-25, 134-135; Foucault, 2009b, 75-77). Pero pensamos también que la cuestión esencial es otra muy distinta. Más interesante que saber si Foucault renuncia por completo al esquema de las disciplinas es evaluar la pertinencia de ese nuevo paradigma de la seguridad para captar los nuevos modos de circulación del poder específicos del tiempo presente, y proceder a un examen detallado de sus atributos: reemplazo de los sistemas de exclusión de lo anormal por la promoción de las prácticas minoritarias, disposición de márgenes amplios de tolerancia, fijación de un marco definido por reglas flexibles de juego en sustitución de la cuadrícula disciplinaria, disposición de una intervención ambiental sobre el medio de acción en el que el individuo opera, establecimiento de la diferencia y la desigualdad, y no de la homogeneidad, como efecto y objetivo fundamental de la acción política, etc.

Algunos pronunciamientos del propio Foucault a finales de los años setenta pueden permitirnos seguir avanzando en esta dirección. En una entrevista concedida en noviembre del año 1977, Foucault habla de un pacto de seguridad que liga en nuestras sociedades al Estado con la población (Foucault, 1994, 385). Este contrato se sitúa al margen de cualquier sistema de legalidad, pues sirve para legitimar intervenciones excepcionales y arbitrarias que la sociedad tolera a cambio de su propia protección. Interrogado acerca de la diferencia entre esta sociedad extra-jurídica en la que vivimos y los regímenes totalitarios, Foucault responde del siguiente modo:

Las sociedades de seguridad que están estableciéndose toleran toda una serie de comportamientos diferentes, variados, en último término incluso desviados y antagónicos los unos con los otros; a condición, es verdad, de que estos se hallen en un cierto envoltorio que elimine las cosas, las personas, los comportamientos considerados como imprevistos [accidentels] y peligrosos. [...] Pero, en este envoltorio, hay un margen de maniobra y un pluralismo tolerados infinitamente más grandes que en el totalitarismo. Es un poder más hábil, más sutil que el del totalitarismo. [...] Es un poder de tipo nuevo (Foucault 1994, 286, trad. nuestra). 
El análisis de este nuevo tipo de poder expresivo del orden de la seguridad es retomado en un coloquio desarrollado en 1979 en la Universidad de Vincennes. Foucault se refiere en este contexto al establecimiento de una economía de poder inédita, alejada del sistema normativo disciplinario, que en la actualidad tiende a articularse en torno a cuatro ejes: señalización de zonas vulnerables, aceptación de un grado considerable de tolerancia, concesión de autonomía a los propios agentes sociales y económicos para la resolución de los conflictos y las hostilidades y, por último, repliegue del poder del Estado salvo en los casos en que la sociedad se vea amenazada por un peligro objetivo (Foucault, 1979).

A pesar de su naturaleza fragmentaria y meramente programática, estas consideraciones de Foucault nos ayudan a pensar de forma adecuada algunos de los aspectos del régimen de poder no disciplinario o "postpanóptico" (Han, 2014; Castro Orellana, 2009) que las técnicas neoliberales de gobierno han puesto en práctica durante los últimos cuarenta años. El debilitamiento del rol del Estado, la desterritorialización y desinstitucionalización de las relaciones de poder como resultado de la globalización política (Deleuze, 1990), la sustitución de los espacios disimétricos de vigilancia por espacios crecientemente interactivos, la utilización política de la libertad del sujeto como instrumento para su propio sometimiento o la promoción, de forma particularmente visible en el ámbito laboral, de valores subjetivos como la autorresponsabilidad, la competitividad, la proactividad, la iniciativa, la creatividad, la disposición a asumir riesgos, la flexibilidad o la capacidad para definir un estilo de vida singular y diferente (Vázquez García, 2005/2006) son sólo algunos de los rasgos definitorios de este poder "más sutil" y "más hábil" que se encuentra vigente en nuestras sociedades. Poner límite a sus pretensiones absolutas exige avanzar en la configuración de renovados modelos de resistencia y de acción política que, para evitar la ineficacia en el plano práctico y la aporía en el teórico, deberán hacerse cargo de los modos diversos en que los individuos, por medio de una libertad que piensan como propia, no son ya simplemente presa inerte de los dispositivos de poder, sino más bien soporte, relevo y función de las distintas declinaciones del poder como gobierno.

\section{Referencias bibliográficas}

BIDET, Jacques (2006): "Foucault et le libéralisme. Rationalité, révolution, résistance", Actuel Marx, 40.

CASTRO ORELLANA, Rodrigo (2006): "Microfísica de la libertad: Foucault y lo político”, Hermenéutica intercultural. Revista de filosofía, 15.

CASTRO ORELLANA, Rodrigo (2009): "La ciudad apestada. Neoliberalismo y postpanóptico", Revista de Ciencia Política, 29.

CASTRO ORELLANA, Rodrigo (2010): "Neoliberalismo y gobierno de la vida", en S. Arribas, G. Cano, J. Ugarte (ed.), Hacer vivir, dejar morir: biopolítica y capitalismo, Madrid, La Catarata.

DARDOT, Pierre ; LAVAL, Christian (2010): La nouvelle raison du monde. Essai sur la société néolibérale, París, La Découverte.

DELEUZE, Gilles (1990): "Post-scriptum sur les sociétés de contrôle", en Pourparlers (1972-1990), París, Éditions de Minuit. 
DÍAZ MARSÁ, Marco (2014): Modificaciones. Ontología crítica y antropología política en el pensamiento de Foucault, Madrid, Escolar y Mayo.

ERIBON, Didier (1992): Michel Foucault, Barcelona, Anagrama.

FOUCAULT, Michel (1979): "Le nouveau contrôle social", en Le nouvel ordre intérieur (Coloquio en la Universidad de Vincennes). Recuperado de: http://www.archives-video. univ paris8.fr/video.php?recordID=111

FOUCAULT, Michel (1988): "El sujeto y el poder", en H. Dreyfus, P. Rabinow (ed.), Michel Foucault: más allá del estructuralismo y la hermenéutica, México, UNAM.

FOUCAULT, Michel (1994): Dits et écrits (vol. III), París, Gallimard.

FOUCAULT, Michel (2000): Un diálogo sobre el poder y otras conversaciones, Madrid, Alianza Editorial.

FOUCAULT, Michel (2003): Hay que defender la sociedad, Madrid, Akal.

FOUCAULT, Michel (2009a): Seguridad, territorio, población, Buenos Aires, Fondo de Cultura Económica.

FOUCAULT, Michel (2009b): Nacimiento de la biopolítica, Madrid, Akal.

FOUCAULT, Michel (2011): La verdad y las formas jurídicas, Barcelona, Gedisa.

FOUCAULT, Michel (2012): Vigilar y castigar: nacimiento de la prisión, Madrid, Siglo XXI ( $1^{\text {a }}$ ed.: 1975).

GARAPON, Antoine (2010): La Raison du moindre État : le néolibéralisme et la justice, París, Odile Jacob.

GARAPON, Antoine (2013): "Michel Foucault, visionnaire du droit contemporain", Raisons politiques, 52 .

GROS, Frédéric (2013): “Introduction”, Raisons politiques, 52.

HACHE, Émilie (2007): "La responsabilité, une technique de gouvernementalité néolibérale ?", Raisons politiques, 28.

HAN, Byung-Chul (2014): Psicopolítica. Neoliberalismo y nuevas técnicas de poder, Barcelona, Herder.

LAGASNERIE, Geoffroy de (2012): La dernière leçon de Michel Foucault. Sur le néolibéralisme, la théorie et la politique, París, Fayard.

LEMKE, Thomas (2004): “"Marx sans guillemets Foucault': Foucault, la gouvernementalité et la critique du néolibéralisme", Actuel Marx, 36.

LÓPEZ ÁLVAREZ, Pablo (2006): "La guerra infinita, el enigma de la sublevación. Michel Foucault y la interpretación bélica de la política”, en N. Sánchez Durá (ed.), La guerra, Valencia, Pre-Textos.

LÓPEZ ÁLVAREZ, Pablo (2010): “Biopolítica, liberalismo y neoliberalismo: acción política y gestión de la vida en el último Foucault", en S. Arribas, G. Cano, J. Ugarte (ed.), Hacer vivir, dejar morir: biopolítica y capitalismo, Madrid, La Catarata.

MORENO PESTAÑA, José Luis (2010): “Gubernamentalidad, biopolítica, neoliberalismo: Foucault en situación”, en S. Arribas, G. Cano, J. Ugarte (ed.), Hacer vivir, dejar morir: biopolítica y capitalismo, Madrid, La Catarata.

MORENO PESTAÑA, José Luis (2011): Foucault y la política, Madrid, Tierradenadie ediciones.

SALAS, Denis (2012): La Justice dévoyée: critique des utopies sécuritaires, París, Éditions des Arènes. 
SARDINHA, Diogo (2005): "La découverte de la liberté", Labyrinthe, 22.

TAYLAN, Ferhat (2013): "L'interventionnisme environnemental, une stratégie néolibérale", Raisons politiques, 52.

VÁZQUEZ GARCÍA, Francisco (2005): “'Empresarios de nosotros mismos’. Biopolítica, mercado y soberanía en la gubernamentalidad neoliberal”, en J. Ugarte (ed.), La administración de la vida. Estudios biopolíticos, Barcelona, Anthropos.

VÁZQUEZ GARCÍA, Francisco (2006): Tras la autoestima. Variaciones sobre el yo expresivo en la modernidad tardía, Donostia-San Sebastián, Gakoa. 